\title{
New encoding schemes for quantum authentication
}

\author{
Priscila García-Fernández ${ }^{1} \quad$ Enrique Fernández-Martínez ${ }^{1} \quad$ Esther Pérez $^{2}$ \\ David J. Santos ${ }^{2}$ \\ ${ }^{1}$ Instituto de Óptica, CSIC, Serrano 123, E-28006 Madrid, Spain \\ ${ }^{2}$ ETSIT, Universidad de Vigo, Campus Universitario s/n, E-36200 Vigo, Spain
}

\begin{abstract}
We study the potential of general quantum operations, Trace-Preserving CompletelyPositive Maps (TPCPs), as encoding and decoding mechanisms in quantum authentication protocols. The study shows that these general operations do not offer significant advantage over unitary encodings. We also propose a practical authentication protocol based on the use of two successive unitary encodings.
\end{abstract}

\section{Introduction}

The past two decades have seen an enormous increase in the development and use of networked and distributed systems, providing increased functionality to the user and a more efficient use of resources. To benefit from such systems, users cooperate by exchanging information over communication channels. In many occasions, this information requires to be protected from unauthorised users. The whole discipline of Cryptography [1] addresses this issue.

Security in communication channels has been greatly improved by recent advances in the field of Quantum Information Processing (QIP). The most notable example is Quantum Key Distribution (QKD) [2, 3], for which the phase of experimental demonstration inside 4] and outside [5, 6] research laboratories has lead to the development of commercial products nowadays. One aspect of QKD that is sometimes underestimated is the dependence of its security on the existence of an authenticated classical channel between the users. Basically, some technique must be designed to allow one party (the verifier) to gain assurances that the identity of another (the claimant) is as declared, thereby preventing impersonation. The most common technique is by the verifier checking the correctness of a message which demonstrates that the claimant is in possession of a secret associated by design with the genuine party. Such a technique is called a Message Authentication Code (MAC), first proposed by Gilbert and co-workers in [7. More formally, an authentication code involves a set of source messages, $\mathcal{S}$, a set of authentication tags, $\mathcal{T}$, a set $\mathcal{K}$ of secret keys, and a set of rules such that each $k \in \mathcal{K}$ defines a mapping to obtain the tag from the source message: $u_{k}: \mathcal{S} \rightarrow \mathcal{T}$. According to this, an authenticated message, consisting of a source state and its tag, $(s, t)$, with $t=u_{k}(s)$, can be verified only by the intended recipient, with whom a key has been shared previously. There are many MACs with different degrees of security. MACs intended to provide unconditional security were first studied by Wegman and Carter [8] combining one-time pads with hash functions. This approach was later pursued by Brassard [9] trading unconditional security for short keys, and further refined by Krawczyk [10].

The use of quantum resources to obtain more efficient classical-message authentication schemes is still an open issue. In [11] the authors showed that quantum information techniques 
can be used to authenticate a binary classical message with a key of length shorter than the one required by classical schemes. However, it is not clear yet whether that would be the case with longer messages. Leung [12] has recognised the potential relevance of authenticating quantum information in future quantum communication systems, and Barnum and coworkers [13] have proposed a secret-key quantum authentication protocol that uses stabiliser purity testing codes. Surprisingly, they showed that any protocol that guarantees secure authenticity must encrypt the quantum plain-text almost perfectly. This fact contrasts with classical MACs, where encryption of the plain-text is not necessary for unconditional security. More recently, Gea-Banacloche [14] has approached data authentication from a steganographic perspective, making use of quantum correcting code techniques.

In this paper we extend and further develop previous work [15, 16] on the authentication of quantum information with unitary coding sets. Our purpose is two-fold. First, in Section 2 we investigate whether the use of reversible TPCP maps in the encoding and decoding stages of our protocol improves its robustness against several common attacks; we restrict ourselves to the simple case of a single qubit since our goal is to compare our results with those of [15]. Second, in Section 3 we study the authentication of arbitrarily complex quantum messages. Now, instead of using a TPCP map, we propose a sequence of two unitary encodings. We show that proceeding in such a way improves the security of the authentication protocol. Finally, in Section 4 we present our main conclusions.

\section{Authentication with general quantum operations}

In the general quantum authentication setting Alice sends a quantum message to Bob with the goal of making Bob confident about the authenticity of such message. If we consider that both participants share a quantum secret key (for example, a set of EPR pairs), and they have access to an authenticated classical channel, then the solution is quite simple: Alice can just use quantum teleportation [17] to send the quantum plain-text. However, here we shall assume that no classical authenticated channel is available, and that the previously shared keys are standard classical bits (the nature of this assumption is practical, given the technological difficulties that still exist to the the manipulation of qubits). Our main goal is to extend the standard MAC technique to the quantum realm. For the sake of simplicity, let us assume that the source quantum message is a qubit described by the density operator $\rho_{\mathcal{S}}$ belonging to a two-dimensional source message space $\mathcal{S}$. Following the standard procedure, Alice appends a tag (a public-known quantum state) to the message in such a way that the recipient, Bob, may verify the tag and so convince himself about the identity of the message originator. The tag is also given by a density operator $\rho_{\mathcal{T}}$, belonging to a tag space $\mathcal{T}$. Therefore, the quantum tagged-message is described by the operator $\rho_{\mathcal{E}}=\rho_{\mathcal{S}} \otimes \rho_{\mathcal{T}}$ that acts on the state space $\mathcal{E}=\mathcal{S} \otimes \mathcal{T}$. To validate the message, Alice and Bob also openly agree on a particular splitting of the tag space $\mathcal{T}$ into valid and invalid tags. The space $\mathcal{T}$ can then be seen as the direct sum of two subspaces, $\mathcal{T}=\mathcal{V} \oplus \mathcal{V}^{\perp}$, where $\mathcal{V}$ is the subspace of valid tags and $\mathcal{V}^{\perp}$ is the subspace of the invalid ones. The splitting of $\mathcal{T}$ leads to the direct sum $\mathcal{E}=\mathcal{C} \oplus \mathcal{C}^{\perp}$, where $\mathcal{C}=\mathcal{S} \otimes \mathcal{V}(\operatorname{dim}(\mathcal{C})=C)$ and $\mathcal{C}^{\perp}=\mathcal{S} \otimes \mathcal{V}^{\perp}$ $\left(\operatorname{dim}\left(\mathcal{C}^{\perp}\right)=D\right)$ are, respectively, the subspaces of valid and invalid tagged messages. A message originally prepared by Alice will thus belong to $\mathcal{C}$.

We shall assume that Alice and Bob share a minimum-size key of just one bit, $k$. Depending on its value, Alice performs an encoding rule on the tagged message: She will apply an $I$ operation in one case (Alice does nothing to the tagged message), and a reversible (to guarantee perfect deterministic decoding) TPCP map [18, 19] in the other. It is worth noting that this is completely equivalent, in security terms, to the use of two TPCP maps. The action of the 
TPCP map on $\rho_{\mathcal{E}}$ can be written as

$$
E\left(\rho_{\mathcal{E}}\right)=\sum_{j} d_{j} U_{j} \rho_{\mathcal{E}} U_{j}^{\dagger}
$$

where the $U_{j}$ are unitary operators, the $d_{j}$ are real and positive, and the following restrictions apply:

$$
\begin{array}{r}
\sum_{j} d_{j}=1, \\
P_{i} U_{k}^{\dagger} U_{j} P_{i}=\delta_{j k},
\end{array}
$$

where $P_{i}$ is the projector onto $\mathcal{C}$. Condition (2) together with the unitarity of the $U_{j}$ operators guarantees that the operation will be trace-preserving. Condition (3) assures the reversibility of the TPCP map if the input state $\rho_{\mathcal{E}}$ belongs to the subspace of valid messages $\mathcal{C}$. After the encoding, Alice will send to Bob, depending on the value of the key, either $\rho_{\mathcal{E}}$ or $E\left(\rho_{\mathcal{E}}\right)$. In both cases we will call the message sent by Alice $\rho_{\mathcal{E}}(k)$. On the reception side, Bob will decode the message sent by Alice according to the value of the the key he also holds: If Alice sent $\rho_{\mathcal{E}}$, Bob will not do anything to the message. Otherwise, he will apply the decoding operation [18, 19]

$$
R\left(E\left(\rho_{\mathcal{E}}\right)\right)=\sum_{j} P_{i} U_{j}^{\dagger} E\left(\rho_{\mathcal{E}}\right) U_{j} P_{i}+P_{N} E\left(\rho_{\mathcal{E}}\right) P_{N},
$$

where $P_{N}=I-\sum_{j} U_{j} P_{i} U_{j}^{\dagger}$. Substituting (11) in (41) we have that $R\left(E\left(\rho_{\mathcal{E}}\right)\right)=P_{i} \rho_{\mathcal{E}} P_{i}=\rho_{\mathcal{E}}$, if $\rho_{\mathcal{E}}$ belongs to $\mathcal{C}$, recovering in this way any valid message sent by Alice. Next Bob has to verify tag appended to the message. If it belongs to $\mathcal{V}$, he will accept the message, otherwise he will discard it suspecting a manipulation in the channel.

In the following subsections we shall analyse the security of this scheme to several attacks. In the forgery attack we shall regard Eve capable of intercepting the state travelling from Alice to Bob, discard it, and forge a new tagged message. In the measurement attack, Eve measures the message in the channel trying to discover the key used. Finally, in the unitary attack, we shall assume that Eve can modify the state in the channel by means of a unitary operation. For simplicity we shall initially consider a two-dimensional tag space $\mathcal{T}$.

\section{$2.1 \quad$ Forgery attack}

Suppose Eve prepares a forged tagged message $\rho_{\mathcal{E}}^{E}$ and sends it to Bob trying to impersonate Alice. When Bob receives this message, depending on the value of the key, he will either apply to the message the decoding operation (4) or he will do nothing. After this decoding, Bob will reject the message if the measurement on the tag space $\mathcal{T}$ shows that it belongs to $\mathcal{V}^{\perp}$. Therefore, the probability $P_{f}$ that Eve deceives Bob is

$$
P_{f}=\frac{1}{2} \operatorname{tr}_{\mathcal{E}}\left[P_{i} \rho_{\mathcal{E}}^{E}+P_{i} R\left(\rho_{\mathcal{E}}^{E}\right)\right]
$$

This quantity depends both on Eve's strategy and on the reverse of the TPCP map actually chosen by Alice and Bob. She will succeed with probability one if

$$
\rho_{\mathcal{E}}^{E} \in \mathcal{C}
$$

and 


$$
R\left(\rho_{\mathcal{E}}^{E}\right)=\sum_{j} P_{i} U_{j}^{\dagger} \rho_{\mathcal{E}}^{E} U_{j} P_{i}+P_{N} \rho_{\mathcal{E}}^{E} P_{N} \in \mathcal{C} .
$$

To better study these conditions we will make use of the following decomposition. Defining the orthogonal projection operators $P_{i}$ and $P_{o}$ as the ones that, respectively, project a state from $\mathcal{E}$ onto $\mathcal{C}$ or onto $\mathcal{C}^{\perp}$, an arbitrary operator $A_{\mathcal{E}}$ can be written as

$$
A_{\mathcal{E}}=A_{i i}+A_{i o}+A_{o i}+A_{o o},
$$

where $A_{j k}=P_{j} A_{\mathcal{E}} P_{k}$, with $j, k=i, o$. If the decomposition (8) is used in operator expressions of the form $\chi_{\mathcal{E}}=A_{\mathcal{E}} \rho_{\mathcal{E}} A_{\mathcal{E}}^{\dagger}$, the corresponding 'i-o' operators are related by the matrix equation

$$
\left(\begin{array}{cc}
\chi_{i i} & \chi_{i o} \\
\chi_{o i} & \chi_{o o}
\end{array}\right)=\left(\begin{array}{cc}
A_{i i} & A_{i o} \\
A_{o i} & A_{o o}
\end{array}\right)\left(\begin{array}{cc}
\rho_{i i} & \rho_{i o} \\
\rho_{o i} & \rho_{o o}
\end{array}\right)\left(\begin{array}{cc}
A_{i i}^{\dagger} & A_{o i}^{\dagger} \\
A_{i o}^{\dagger} & A_{o o}^{\dagger}
\end{array}\right),
$$

where $A_{j k}^{\dagger}=P_{k} A_{\mathcal{E}}^{\dagger} P_{j}$.

Using this decomposition, condition (6) requires the following matrix form for $\rho_{\mathcal{E}}^{E}$ :

$$
\rho_{\mathcal{E}}^{E}=\left(\begin{array}{cc}
\rho_{\mathcal{S}}^{E} & 0 \\
0 & 0
\end{array}\right)
$$

In condition (77), the summation in the right side is always in $\mathcal{C}$ due to the action of $P_{i}$. We are left with the second term. Writing $U_{j}=U_{i i}^{j}+U_{i o}^{j}+U_{o i}^{j}+U_{o o}^{j}$, where $U_{k l}^{j}=P_{k} U_{j} P_{l}$, we have:

$$
P_{N}=I-\sum_{j} U_{j} P_{i} U_{j}^{\dagger}=\left(\begin{array}{cc}
I & 0 \\
0 & I
\end{array}\right)-\sum_{j}\left(\begin{array}{cc}
U_{i i}^{j} U_{i i}^{j \dagger} & U_{i i}^{j} U_{o i}^{j \dagger} \\
U_{o i}^{j} U_{i i}^{j \dagger} & U_{o i}^{j} U_{o i}^{j \dagger}
\end{array}\right) \equiv\left(\begin{array}{cc}
I & 0 \\
0 & I
\end{array}\right)-\left(\begin{array}{cc}
G_{i i} & H \\
H^{\dagger} & G_{o i}
\end{array}\right) .
$$

With this notation the second term of (7) vanishes when

$$
H^{\dagger} \rho_{\mathcal{S}}^{E} H=0
$$

and

$$
\left(G_{i i}-I\right) \rho_{\mathcal{S}}^{E} H=0,
$$

where $H=\sum_{j} U_{i i}^{j} U_{o i}^{j \dagger}$, and $G_{i i}=\sum_{j} U_{i i}^{j} U_{i i}^{j \dagger}$. In order to further analyse these equations let us first study the structure of the $U_{j}$ operators. Since the tag space is two-dimensional, each $U_{j}$ will be a $4 \times 4$ square matrix. Given their unitarity, their rows and columns will be orthonormal vectors. We may write two of these operators in column-vector form as

$$
\begin{gathered}
U_{1}=\left(u_{1}\left|u_{2}\right| u_{3} \mid u_{4}\right), \\
U_{2}=\left(v_{1}\left|v_{2}\right| v_{3} \mid v_{4}\right),
\end{gathered}
$$

where the sets $\left\{u_{j}\right\}$ and $\left\{v_{j}\right\}$ are two basis of a four-dimensional vectorial space. The reversibility condition, Eq. (3), imposes that the first two vectors of each set must also be orthogonal, so the set $\left\{u_{1}, u_{2}, v_{1}, v_{2}\right\}$ will also be a base. This means that we can only have two operators composing the TPCP map. A third one will necessarily have its first two columns orthogonal to the first two columns of the other two operators, which is impossible. Therefore, our encoding operation can only be composed of up to $\operatorname{dim}(\mathcal{E}) / \operatorname{dim}(\mathcal{C})(2$ in our case) unitary operators. 
With two unitary operators forming the encoding operation and a two-dimensional tag space, we can calculate $H=\sum_{j} U_{i i}^{j} U_{o i}^{j \dagger}$. Using the fact that $\left\{u_{1}, u_{2}, v_{1}, v_{2}\right\}$ is an orthonormal base, we obtain $H=0$, and analogously $G_{i i}=\sum_{j} U_{i i}^{j} U_{i i}^{j \dagger}=I$. So Eve will always fulfil conditions (12) and (13). This will always happen if we choose the maximum number of unitary operators allowed by the dimensions of $\mathcal{E}$ and $\mathcal{C}$. One possibility to overcome this would be to choose only one unitary operator, but then the encoding operation would be unitary, and it has been shown in [15] that with a one-bit key a unitary encoding is not secure. Another possibility would be to use a larger tag space $\mathcal{T}$ that will increment $\operatorname{dim}(\mathcal{E})$ without increasing $\operatorname{dim}(\mathcal{C})(\mathcal{V}$ will remain as a one-dimensional subspace of $\mathcal{T}$ ). This will allow us to use more unitary operators to form the non-unitary TPCP map without choosing the maximum of operators allowed by the dimensions of $\mathcal{E}$ and $\mathcal{C}$. Specifically, if we use two qubits for the tag, we have a four-dimensional tag space $\mathcal{T}$. Imposing that the valid tag subspace $\mathcal{V}$ is one-dimensional, we have that $\operatorname{dim}(\mathcal{E})=8, \operatorname{dim}(\mathcal{C})=2$, and $\operatorname{dim}(\mathcal{E}) / \operatorname{dim}(\mathcal{C})=4$. We can thus choose up to three unitary operators to construct the TPCP map. With two or three operators it is easy for Alice and Bob to make impossible for Eve to satisfy (12) and (13). This follows from the fact that to fulfil (12) Eve has to choose $\rho_{\mathcal{S}}^{E}$ with support on the kernel of $H^{\dagger}$. But since since $\operatorname{dim}(\mathcal{C})<\operatorname{dim}\left(\mathcal{C}^{\perp}\right)$ and $H^{\dagger}$ acts from $\mathcal{C}$ on $\mathcal{C}^{\perp}$, Alice and Bob can always choose $U_{j}$ such that the kernel of $H^{\dagger}$ is empty. Therefore, there is no $\rho_{\mathcal{S}}^{E}$ fulfilling (12). Therefore, in the following we will shall consider a tag space of dimension greater than two.

\subsection{Measurement attack}

Now Eve tries to measure the message sent by Alice to discover the key used and replace the message with one of her own. Since Eve does not know which message, $\rho_{\mathcal{S}}$, was sent, the only way she has to discern between the two possible values of the key is measuring the tag. Therefore, to make this strategy successful, Eve needs the tag of any message encoded with $E(\cdot)$ to belong to $\mathcal{V}^{\perp}$ so as to distinguish it from the tag of a message encoded with $I$ that belongs to $\mathcal{V}$. Specifically, Eve needs that

$$
\sum_{j} d_{j}\left(\begin{array}{cc}
U_{i i}^{j} & U_{i o}^{j} \\
U_{o i}^{j} & U_{o o}^{j}
\end{array}\right)\left(\begin{array}{cc}
\rho_{\mathcal{S}} & 0 \\
0 & 0
\end{array}\right)\left(\begin{array}{cc}
U_{i i}^{j \dagger} & U_{o i}^{j \dagger} \\
U_{i o}^{j \dagger} & U_{o o}^{j \dagger}
\end{array}\right)=\left(\begin{array}{cc}
0 & 0 \\
0 & \rho_{\mathcal{S}}^{\prime}
\end{array}\right) .
$$

This condition requires that

$$
\sum_{j} d_{j} U_{i i}^{j} \rho_{\mathcal{S}} U_{i i}^{j \dagger}=0, \quad \forall \rho_{\mathcal{S}}
$$

and

$$
\sum_{j} d_{j} U_{o i}^{j} \rho_{\mathcal{S}} U_{i i}^{j \dagger}=0, \quad \forall \rho_{\mathcal{S}} .
$$

Since the $d_{j}$ are positive, each term in (17) is positive and Eve needs that

$$
U_{i i}^{j} \rho_{\mathcal{S}} U_{i i}^{j \dagger}=0, \quad \forall \rho_{\mathcal{S}}, \forall j .
$$

This is true $\forall \rho_{\mathcal{S}}$ iff $U_{i i}^{j}=0, \forall j$, which is easily avoided by design.

\subsection{Unitary attack}

Let us assume now that Eve performs a unitary quantum operation $F_{\mathcal{E}}$ on the encoded tagged message in transit between Alice and Bob. This operation changes the state of the encoded 
message from $\rho_{\mathcal{E}}(k)$ to $F_{\mathcal{E}} \rho_{\mathcal{E}}(k) F_{\mathcal{E}}^{\dagger}$. Bob, ignorant about this action, will perform his decoding operation on the encoded tagged message received. Since Eve does not know the value of the key used, the probability of deceiving Bob will be

$$
P_{u}=\frac{1}{2} \operatorname{tr}_{\mathcal{E}}\left[P_{i} F_{\mathcal{E}} \rho_{\mathcal{E}} F_{\mathcal{E}}^{\dagger}+P_{i} R\left(F_{\mathcal{E}} E\left(\rho_{\mathcal{E}}\right) F_{\mathcal{E}}^{\dagger}\right)\right]
$$

with

$$
R\left(F_{\mathcal{E}} E\left(\rho_{\mathcal{E}}\right) F_{\mathcal{E}}^{\dagger}\right)=\sum_{k} P_{i} U_{k}^{\dagger} F_{\mathcal{E}} \sum_{j} d_{j} U_{j} \rho_{\mathcal{E}} U_{j}^{\dagger} F_{\mathcal{E}}^{\dagger} U_{k} P_{i}+P_{N} F_{\mathcal{E}} \sum_{j} d_{j} U_{j} \rho_{\mathcal{E}} U_{j}^{\dagger} F_{\mathcal{E}}^{\dagger} P_{N}
$$

In other words, for Eve to succeed with $P_{u}=1$, she needs that

$$
F_{\mathcal{E}} \rho_{\mathcal{E}} F_{\mathcal{E}}^{\dagger} \in \mathcal{C}
$$

and

$$
R\left(F_{\mathcal{E}} E\left(\rho_{\mathcal{E}}\right) F_{\mathcal{E}}^{\dagger}\right) \in \mathcal{C}
$$

The first condition means that

$$
\left(\begin{array}{ll}
F_{i i} & F_{i o} \\
F_{o i} & F_{o o}
\end{array}\right)\left(\begin{array}{cc}
\rho_{\mathcal{S}} & 0 \\
0 & 0
\end{array}\right)\left(\begin{array}{ll}
F_{i i}^{\dagger} & F_{o i}^{\dagger} \\
F_{i o}^{\dagger} & F_{o o}^{\dagger}
\end{array}\right)=\left(\begin{array}{cc}
\rho_{\mathcal{S}}^{\prime} & 0 \\
0 & 0
\end{array}\right) .
$$

This condition is satisfied if

$$
F_{\mathcal{E}}=\left(\begin{array}{cc}
F_{i i} & 0 \\
0 & F_{o o}
\end{array}\right)
$$

Writing (23) as in (21), we see that the first term of (21) always fulfils (23). Suppose Eve can choose the operator $F_{\mathcal{E}}$ such that it commutes with $P_{N}$, then the second term in (21) transforms to

$$
F_{\mathcal{E}} P_{N} \sum_{j} d_{j} U_{j} P_{i} \rho_{\mathcal{E}} P_{i} U_{j}^{\dagger} P_{N} F_{\mathcal{E}}^{\dagger}
$$

where we have also used the fact that $\rho_{\mathcal{E}}$ belongs to $\mathcal{C}$, and so $\rho_{\mathcal{E}}=P_{i} \rho_{\mathcal{E}} P_{i}$. Now using the reversibility condition (3) it is easy to see that the operator $P_{N} U_{j} P_{i}$ is identically zero for all $j$ :

$$
P_{N} U_{j} P_{i}=\left(I-\sum_{k} U_{k} P_{i} U_{k}^{\dagger}\right) U_{j} P_{i}=U_{j} P_{i}-\sum_{k \neq j} U_{k} P_{i} U_{k}^{\dagger} U_{j} P_{i}-U_{j} P_{i} U_{j}^{\dagger} U_{j} P_{i}=0,
$$

where the second term is zero because of (3), and the other two terms cancel out each other. Thus we have shown that, provided Eve can find a unitary operator $F_{\mathcal{E}}$ fulfilling (25) and commuting with the projector $P_{N}$, she can deceive Bob with probability equal to one. Does such unitary operator exist?

Let us explore the similarity of this problem to the one we faced in [16] (unitary encodings). Let $P_{M}$ be the the projector orthogonal to $P_{N}$ :

$$
P_{M}=I-P_{N}=\sum_{j} U_{j} P_{i} U_{j}^{\dagger}=\left(\begin{array}{cc}
G_{i i} & H \\
H^{\dagger} & G_{o i}
\end{array}\right) .
$$


Since $F_{\mathcal{E}}$ is unitary, condition (25) is equivalent to $\left[F_{\mathcal{E}}, P_{i}\right]=0$, and so our problem (Eve finding $F_{\mathcal{E}}$ making $P_{u}=1$ ) can be stated in compact form as:

$$
\begin{aligned}
& {\left[F_{\mathcal{E}}, P_{i}\right]=0,} \\
& {\left[F_{\mathcal{E}}, P_{M}\right]=0 .}
\end{aligned}
$$

Note that these equations do not explicitly depend on the index $j$, that is, on the individual form of the encoding operators $U_{j}$, but on a global characteristic of the TPCP map (through the sum inside $P_{M}$ ). For this individual dependence to dissappear, and also for $P_{M}$ to be a projector, it is essential the relation between the $U_{j}$ defined by the reversibility condition.

From [16] we know that the problem given by Eqs. (30)-(31) has solution regardless its dimension and the explicit form or rank of the two projectors. In fact, the problem is completely equivalent to the one studied there for the case of a unitary encoding with just one bit of key. In particular, the family of $F_{\mathcal{E}}$ fulfilling (30)-(31) is the same, and so Eve can transform the original message as desired. Thus we conclude that, using the more general set of reversible TPCP maps for encoding, one bit of key is still not enough to authenticate one qubit quantum message.

\section{Double encoding}

In the preceding section we have shown that using more general encodings, such as TPCP maps, does not provide any advantage over unitary operations in the authentication of an elemental piece of quantum information (a qubit). Therefore, it seems reasonable to undertake the authentication of arbitrary quantum information with unitary encodings. In [16] we did so for the case in which Alice and Bob share a classical $n$-bit key. According to this authentication procedure, Alice, depending on the value of the key, performs a unitary encoding rule on the tagged message, $U(k)$, selected from the unitary coding set $\{U(0), \cdots, U(K-1)\}$, where $K=2^{n}$ and $U(0)=I$. We found the necessary conditions that this unitary coding set must satisfy to protect against forgery and unitary attacks. Unfortunately, these conditions cannot be fulfilled simultaneously, i.e. the encoding operators that minimise Eve's probability of success in a forgery attack does not protect at all against the unitary attack. In this section we present a two-stage encoding procedure that tries to circumvent such deficiency. The different phases of this new protocol are the following:

1. Tag 1: Alice prepares her message, $\rho_{\mathcal{S}}$, which belongs to the state space $\mathcal{S}(\operatorname{dim}(\mathcal{S})=S)$. She now appends to the message a tag described by $\rho_{\mathcal{T}_{1}}$ that belongs to the state space $\mathcal{T}_{1}\left(\operatorname{dim}\left(\mathcal{T}_{1}\right)=T_{1}\right)$. The space of tagged messages is $\mathcal{E}_{1}=\mathcal{S} \otimes \mathcal{T}_{1}$, and the tagged message will be described by $\rho_{\mathcal{E}_{1}}=\rho_{\mathcal{S}} \otimes \rho_{\mathcal{T}_{1}}$. Alice and Bob openly agree on a particular splitting of the tag space $\mathcal{T}_{1}$ into the direct sum of two subspaces $\mathcal{T}_{1}=\mathcal{V}_{1} \oplus \mathcal{V}_{1}^{\perp}$, where $\mathcal{V}_{1}$ is the subspace of valid tags, and $\mathcal{V}_{1}^{\perp}$ is the subspace of the invalid ones. This splitting of $\mathcal{T}_{1}$ leads to the direct sum $\mathcal{E}_{1}=\mathcal{C}_{1} \oplus \mathcal{C}_{1}^{\perp}$, where $\mathcal{C}_{1}=\mathcal{S} \otimes \mathcal{V}_{1}$ is the subspace of valid messages $\left(\operatorname{dim}\left(\mathcal{C}_{1}\right)=C_{1}\right)$, and $\mathcal{C}_{1}^{\perp}=\mathcal{S} \otimes \mathcal{V}_{1}^{\perp}$ is the subspace of the invalid ones $\left(\operatorname{dim}\left(\mathcal{C}_{1}^{\perp}\right)=D_{1}\right)$.

2. Encoding 1: Alice, depending on the value of a $n_{1}$-bit key shared with Bob, performs an encoding rule on the tagged message. The encoding rule, $U_{1}(p)$, is selected from the unitary encoding set $\left\{U_{1}(0), \cdots, U_{1}\left(K_{1}-1\right)\right\}$, where $K_{1}=2^{n_{1}}$ and $U_{1}(0)=I$. If the tagged message is $\rho_{\mathcal{E}_{1}}$, the state of the message after this encoding is

$$
\rho_{\mathcal{E}_{1}}(p)=U_{1}(p) \rho_{\mathcal{E}_{1}} U_{1}^{\dagger}(p) .
$$


Thus, a tagged message encoded by Alice with $U_{1}(p)$ will necessarily belong to $\mathcal{C}_{p}$, the subspace of all the tagged messages transformed by $U_{1}(p)$. The aim of this first encoding is to protect against unitary attacks. As it has been shown in [16], the $\mathcal{C}_{k}$ subspaces, $k=$ $0, \ldots, K_{1}-1$, must be chosen at an intermediate point between zero and total overlapping so as to offer the best possible protection against this type of attack.

3. Tag 2: After this first encoding, Alice appends a second tag $\rho_{\mathcal{T}_{2}}$ to the already encoded and tagged message, obtaining

$$
\rho_{\mathcal{E}}=\rho_{\mathcal{E}_{1}}(p) \otimes \rho_{\mathcal{T}_{2}} .
$$

The state space of the second tag is $\mathcal{T}_{2}$ and is analogously divided into the subspaces of valid and invalid tags $\mathcal{T}_{2}=\mathcal{V}_{2} \oplus \mathcal{V}_{2}^{\perp}$. The space of the messages tagged twice is $\mathcal{E}=\mathcal{E}_{1} \otimes \mathcal{T}_{2}=\mathcal{S} \otimes \mathcal{T}_{1} \otimes \mathcal{T}_{2}$, and the valid and invalid message subspaces $\mathcal{E}=\mathcal{C} \oplus \mathcal{C}^{\perp}$ $\left(\operatorname{dim}(\mathcal{C})=C, \operatorname{dim}\left(\mathcal{C}^{\perp}\right)=D\right)$. The second tag appended by Alice will thus belong to $\mathcal{V}_{2}$. Regardless of the dimension of $\mathcal{T}_{2}$, we shall assume $\mathcal{V}_{2}$ to be one-dimensional, with $|0\rangle_{\mathcal{T}_{2}}$ a base of this subspace, and thus $\rho_{\mathcal{T}_{2}}=|0\rangle\left\langle\left. 0\right|_{\mathcal{T}_{2}}\right.$.

4. Encoding 2: Finally, Alice performs a second encoding on the twice-tagged message depending on the value of a second key $q$ shared with Bob. The encoding rule, $U_{2}(q)$, is selected from the unitary coding set $\left\{U_{2}(0), \cdots, U_{2}\left(K_{2}-1\right)\right\}$, with $U_{2}(0)=I$. This second encoding is intended to protect the message against forgery attacks without interfering with the first encoding. In order to achieve this goal, Alice and Bob choose a number of operators $K_{2}-1$ equal to the dimension of the invalid tag subspace $\mathcal{V}_{2}^{\perp}$. Let us consider the following base of this subspace: $\left\{|1\rangle_{\mathcal{T}_{2}},|2\rangle_{\mathcal{T}_{2}}, \ldots,\left|K_{2}-1\right\rangle_{\mathcal{T}_{2}}\right\}$. The encoding operators $\left\{U_{2}(0), \cdots, U_{2}\left(K_{2}-1\right)\right\}$ acting on $\mathcal{E}$ can be written as $U_{2}(k)=\sum_{j, l=0}^{K_{2}-1} U_{2}(k)_{j l} \otimes|j\rangle\left\langle\left. l\right|_{\mathcal{T}_{2}}\right.$ with $U_{2}(k)_{j l}$ acting on $\mathcal{E}_{1}=\mathcal{S} \otimes \mathcal{T}_{1}$. We shall further impose on the unitary encoding operators the condition $U_{2}(k)_{0 j}=U_{2}(k)_{j 0}=\delta_{j k}$. As we shall explain later, this allows to protect the message against forgery without interfering with the first encoding. After this second encoding, the expression for the message to be sent through the channel is:

$$
\rho_{\mathcal{E}}(q)=U_{2}(q) \rho_{\mathcal{E}_{1}}(p) \otimes|0\rangle\left\langle\left. 0\right|_{\mathcal{T}_{2}} U_{2}^{\dagger}(q)\right.
$$

5. Verification: When Bob receives the message, he performs the matching decoding rule to Alice's second encoding, $U_{2}^{\dagger}(q)$, and measures the second tag attached. If it belongs to $\mathcal{V}_{2}$, he will continue, after tracing out the second tag attached, with the verification procedure:

He will first decode, with $U_{1}^{\dagger}(p)$, Alice's first encoding, and then will measure the first tag and check whether it belongs to $\mathcal{V}_{1}$. If this is the case, Bob will regard the message as authentic and will recover the original plain-text after tracing out both tags.

We shall now analyse the effects of Eve's attacks on this protocol.

\subsection{Forgery attack}

In this attack Eve has the power to replace the tagged message in transit between Alice and Bob with a forged tagged message of her own, $\rho_{E}$. From this message, Bob will perform his first decoding obtaining

$$
U_{2}^{\dagger}(q) \rho_{E} U_{2}(q)
$$


with $q$ the value of the second key shared with Alice. The probability of Eve being undetected is

$$
P_{f}=\frac{1}{K_{2}} \sum_{k=0}^{K_{2}-1} \operatorname{tr}_{\mathcal{E}}\left[P_{2} U_{2}(k)^{\dagger} \rho_{E} U_{2}(k)\right]=\frac{1}{K_{2}} \sum_{k=0}^{K_{2}-1} \operatorname{tr}_{\mathcal{E}}\left[P_{2}(k) \rho_{E}\right],
$$

where $P_{2}=I_{\mathcal{E}_{1}} \otimes|0\rangle\left\langle\left. 0\right|_{\mathcal{T}_{2}}\right.$ is the projector onto $\mathcal{C}$, and $P_{2}(k)=U_{2}(k) P_{2} U_{2}^{\dagger}(k)$ is the projector onto $\mathcal{C}$ transformed by $U_{2}(k)$. But, since we have imposed before that $U_{2}(k)_{0 j}=U_{2}(k)_{j 0}=\delta_{j k}$, $P_{2}(k)=I_{\mathcal{E}_{1}} \otimes|k\rangle\left\langle\left. k\right|_{\mathcal{T}_{2}}\right.$, and then $P_{f}=1 / K_{2}$. Therefore, Alice and Bob can decrease Eve's success probability increasing $K_{2}$, protecting in this way the message against forgery. Note also that the projectors $P_{2}(k)$ are mutually orthogonal. In other words, the effect of the condition imposed over the set of operators $U_{2}(k)$ is to transform the space of valid messages $\mathcal{C}$ into orthogonal, disjoint subspaces. In [16] we showed that this type of unitary set provides optimal protection against forgery attacks.

\subsection{Unitary attack}

Let us now assume that Eve performs a unitary quantum operation $F_{\mathcal{E}}$ on the encoded tagged message in transit between Alice and Bob. This operation changes the state of the message from $\rho_{\mathcal{E}}(q)$ to $F_{\mathcal{E}} \rho_{\mathcal{E}}(q) F_{\mathcal{E}}^{\dagger}$. Bob, ignorant about this action, will perform his first decoding operation on the message received, obtaining as the decoded tagged message

$$
U_{2}^{\dagger}(q) F_{\mathcal{E}} \rho_{\mathcal{E}}(q) F_{\mathcal{E}}^{\dagger} U_{2}(q)
$$

Thus, the probability of Eve being unnoticed after this first test is

$$
P_{u}=\frac{1}{K_{2}} \sum_{k=0}^{K_{2}-1} \operatorname{tr}_{\mathcal{E}}\left[P_{2} U_{2}^{\dagger}(k) F_{\mathcal{E}} \rho_{\mathcal{E}}(k) F_{\mathcal{E}}^{\dagger} U_{2}(k)\right]=\frac{1}{K_{2}} \sum_{k=0}^{K_{2}-1} \operatorname{tr}_{\mathcal{E}}\left[F_{\mathcal{E}}^{\dagger} P_{2}(k) F_{\mathcal{E}} \rho_{\mathcal{E}}(k)\right]
$$

Since the subspaces obtained after transforming $\mathcal{C}$ with the set $U_{2}(k)$ are all orthogonal, disjoint subspaces of $\mathcal{E}$, if Eve chooses a unitary operator $F_{\mathcal{E}}$ acting inside each subspace separately according to

$$
F_{\mathcal{E}}=\sum_{j=0}^{K_{2}-1} F_{j j} \otimes|j\rangle\left\langle\left. j\right|_{\mathcal{T}_{2}}\right.
$$

with $F_{j j}$ unitary and acting on $\mathcal{E}_{1}$, then $F_{\mathcal{E}}^{\dagger} P_{2}(k) F_{\mathcal{E}}=P_{2}(k)$, and she will always pass Bob's first test. The manipulated message that Bob accepts after his first test will be

$$
\rho_{\mathcal{E}_{1}}^{\prime}(p)=\operatorname{tr}_{\mathcal{T}_{2}}\left(P_{2}(q) F_{\mathcal{E}} U_{2}(q) \rho_{\mathcal{E}_{1}}(p) \otimes|0\rangle\left\langle\left. 0\right|_{\mathcal{T}_{2}} U_{2}^{\dagger}(q) F_{\mathcal{E}}^{\dagger}\right) .\right.
$$

After some algebra it can be shown that $\rho_{\mathcal{E}_{1}}^{\prime}(p)$ reduces to $F_{q q} \rho_{\mathcal{E}_{1}}(p) F_{q q}^{\dagger}$, where $\rho_{\mathcal{E}_{1}}(p)$ is the original message tagged for the first time by Alice, and encoded with $U_{1}(p)$. Bob will now decode this message with $U_{1}^{\dagger}(p)$, and verify the first tag appended by Alice. Therefore, if Alice and Bob choose the unitary encoding set $\left\{U_{1}(0), \cdots, U_{1}\left(K_{1}-1\right)\right\}$ to offer the best possible protection against a unitary attack regardless of its effectiveness against a forgery attack, the entire procedure will protect the message simultaneously against both attacks with the best protection that a single encoding can give against each attack separately. 


\section{Conclusion}

In Section 2 our goal has been to investigate whether the use of TPCP maps in the encoding and decoding operations could improve the security of authentication protocols as compared to the unitary encoding studied in [15] and [16. In [15] we showed that one bit of key is enough to protect one qubit of message against forgery or measurement attacks, but not against unitary attacks. One could expect that the extended complexity of a TPCP encoding would protect more efficiently against these attacks. However, we have found exactly the opposite to hold: the use of a TPCP map does not improve at all the security of the protocol with one bit of key; in fact, there is no reason for Alice and Bob to use it, as it needs more resources. This result is strongly related to the reversibility condition imposed on the TPCP map. That condition requires the TPCP map to act inside the coding subspace, effectively, as a collective unitary operator.

With this idea in mind, in Section 3 we have approached the authentication of arbitrary quantum information with a double encoding. We have shown that it offers simultaneous protection against unitary and forgery attacks with the best protection that a single encoding can give to each attack separately.

Many open questions related to the quantum authentication schemes analysed deserve further investigation. For example, a more practical definition of the probability of failure is required. For instance, Eve might, with high probability, transform the original message, but only in a way such that the fidelity between the original and the transformed state be still very high. It could also be the case that she could strongly transform, without being noticed, messages inside a particular subspace of the valid message space, and be noticed if she transforms messages outside of it. All these situations would have to be considered by Alice and Bob in any practical implementation of the protocol.

\section{Acknowledgements}

The authors acknowledge stimulating discussions with M. Curty. This work was partially supported by the Spanish Government (grant Nos. TIC1999-0645-C05-03, BFM2000-0806 and TIC2001-3217), by Comunidad de Madrid (Spain, grant No. 07T/0063/2000) and Xunta de Galicia (Spain, grant No. PGIDT00PXI322060PR).

\section{References}

[1] A. J. Menezes, P. C. Van Oorschot, and S. A. Vanstone. Handbook of Applied Cryptography. Series on Discrete Mathematics and Its Applications. CRC Press, 1996.

[2] C. H. Bennett and G. Brassard. Quantum cryptography: Public key distribution and coin tossing. In Proceedings of IEEE International Conference on Computers, Systems and Signal Processing, pages 175-179, New York, december 1984. IEEE Press.

[3] A. K. Ekert. Quantum cryptography based on Bell's theorem. Physical Review Letters, 67(6):661-663, august 1991.

[4] C. H. Bennett and J. Smolin. Experimental quantum cryptography. Journal of Cryptology, 5(1):3-28, 1992.

[5] A. Muller, H. Zbinden, and N. Gisin. Underwater quantum coding. Nature, 378:449, november 1995. 
[6] A. Muller, H. Zbinden, and N. Gisin. Quantum cryptography over $23 \mathrm{~km}$ in installed under-lake telecom fibre. Europhysics Letters, 33:335-339, 1996.

[7] E. N. Gilbert, F. J. MacWillians, and N. J. A. Sloane. Codes which detect deception. Bell System Technical Journal, 53:405-424, 1974.

[8] M. N. Wegman and J. L. Carter. New hash functions and their use in authentication and set equality. Journal of Computer and System Sciences, 22:265-279, 1981.

[9] G. Brassard. On computationally secure authentication tags requiring short secret shared keys. Advances in Cryptology - Proceedings of Crypto 82, pages 79-86, 1983.

[10] H. Krawczyk. LFSR-based hashing and authentication. Advances in Cryptology - Proceedings of Crypto 94, pages 129-139, 1994.

[11] M. Curty and D. J. Santos. Quantum authentication of classical messages. Physical Review A, 64:062309, 2001.

[12] D. W. Leung. Quantum Vernam cipher. Quantum Information $\&$ Computation, 2(1):14-34, 2002 .

[13] H. Barnum, C. Crépeau, D. Gottesman, A. Smith, and A. Tapp. Authentication of quantum messages. quant-ph/0205128.

[14] J. Gea-Banacloche. Hiding messages in quantum data. Journal of Mathematical Physics, 43(9):4531-4536, 2002.

[15] M. Curty, D. J. Santos, E. Pérez, and P. García-Fernández. Qubit authentication. Physical Review A, 66:022301, 2002.

[16] E. Pérez, M. Curty, D. J. Santos, and P. García-Fernández. Quantum authentication with unitary coding sets. Journal of Modern Optics, 50(6-7):1035-1047, 2003.

[17] C. H. Bennett, G. Brassard, C. Crépeau, R. Jozsa, A. Peres, and W.K. Wootters. Teleporting an unknown quantum state via dual classical and Einstein-Podolsky-Rosen channels. Physical Review Letters, 70:1895-1899, march 1993.

[18] M. A. Nielsen, C. M. Caves, B. Schumacher, and H. Barnum. Information-theoretic approach to quantum error correction and reversible measurement. Proc. R. Soc. Lond. A, 454:277-304, 1998.

[19] C. M. Caves. Quantum error correction and reversible operations. Journal of Superconductivity, 12(6):707-718, 1999. 